\title{
El HÁBITO y LA FORMA de CRECIMIENTO EN LA TRIBU CACTEAE (CACtaceae, Cactoideae)
}

\author{
Monserrat Vázquez-Sánchez ${ }^{1,3}$, Teresa Terrazas ${ }^{1}$ y Salvador Arias ${ }^{2}$ \\ ${ }^{1}$ Departamento de Botánica, Instituto de Biología, Universidad Nacional Autónoma de México \\ ${ }^{2}$ Jardín Botánico, Instituto de Biología, Universidad Nacional Autónoma de México \\ ${ }^{3}$ Autor para la correspondencia: monsevaz@ibiologia.unam.mx
}

\begin{abstract}
Resumen: Los términos 'forma de crecimiento' y 'hábito' con frecuencia se usan como sinónimos; sin embargo, su asignación a las diferentes especies resulta problemática en algunos grupos taxonómicos debido a la diversidad morfológica; éste es el caso en Cactaceae, particularmente en la tribu Cacteae de la subfamilia Cactoideae. Se estudió la morfología del tallo de 102 especies (26 géneros) de Cacteae con el objetivo de identificar el hábito y reconocer cuántas formas de crecimiento se presentan en la tribu, así como para hacer una distinción clara entre los términos 'hábito' y ‘forma de crecimiento' para Cactaceae y discutir sus diferencias respecto a los conceptos de forma de vida y arquitectura vegetal. Con base en las observaciones y mediciones para 102 especies de Cacteae se reconocieron cuatro formas de crecimiento (cilíndrica, columnar, globosa y globosa-deprimida). Por su tamaño y longevidad no es posible utilizar los términos 'árbol' o 'hierba' para las especies de Cacteae. El 12\% de las especies estudiadas de esta tribu tienen ramificación basítona (arbustos) como Acharagma roseana, Ferocactus pilosus y Thelocactus bicolor. Sugerimos utilizar únicamente el término 'forma crecimiento' para referirse a los tallos de la tribu Cacteae con la finalidad de evitar confusiones. Palabras clave: arbusto, arquitectura vegetal, cilíndrica, columnar, forma de vida, globoso.
\end{abstract}

\begin{abstract}
The terms 'growth form' and 'habit' are often used as synonyms. However, their assignment to different species is problematic because of the morphological diversity occurring in some taxonomic groups, as is the case in Cactaceae, particularly in the tribe Cacteae of the Cactoideae subfamily. Stem morphology was studied in 102 species (26 genera) of Cacteae in order to identify the habit and to recognize how many growth forms occur in the tribe, as well to make a distinction between the concepts of habit and growth form in Cactaceae, and to discuss the differences between them and the concepts of life form and plant architecture. Based on observations and measurements for 102 species of Cacteae, four growth forms (cylindrical, columnar, globose, and globose-depressed) were recognized. Neither the habit 'tree' or 'herb' can be assigned to any member of Cacteae based on their size or longevity. Twelve percent of the studied species of this tribe have basitonic branching (shrubs) as for Acharagma roseana, Ferocactus pilosus and Thelocactus bicolor. In order to avoid confusion, we suggest using only the term 'growth form' when referring to the various stem forms in the Cacteae tribe.
\end{abstract}

Keywords: columnar, cylindric, globose, life form, plant architecture, shrub.

$\mathbf{E}_{\mathrm{p} e \boldsymbol{s}}$ término hábito, del latín habitus, significa porte o aspecto exterior (Font Quer, 1977). Harris y Harris (2001) definen 'hábito' como la apariencia general o el modo de crecimiento de una planta. Para Judd et al. (2002) este término denota la apariencia general de una planta y consideran tres hábitos: (1) árbol (con un tronco bien definido), (2) arbusto (varias ramas que surgen en la posición basal y más bajo que un árbol), ambos con tejido leñoso (lignificados), y (3) hierba (tallos anuales no lignificados). Los términos 'árbol', 'arbusto' y 'hierba' son el antecedente más antiguo de clasificación propuesto por Teofrasto (300 A.C.) con base en la forma de las plantas (Díaz et al., 2002).
'Forma de crecimiento' y 'forma de vida' se usan frecuentemente como sinónimos de 'hábito' al referirse a la forma de una planta (Mueller-Dombois y Ellenberg, 1974; Moreno, 1984; Pavón et al., 2000). Sin embargo, el término 'forma de vida' fue propuesto por Raunkiaer (1934) para clasificar a las plantas con base en la posición de las yemas u órganos de renuevo, a partir de los cuales se desarrollarán nuevos brotes o nuevo follaje después de la época desfavorable. Ejemplos de formas de vida según Raunkiaer son 'criptófita' y 'geófita', y estos términos tienen una connotación adaptativo-ecológica (Mueller-Dombois y Ellenberg, 1974). Por el contrario, ‘forma de crecimiento' describe el 
diseño o figura y la construcción de los individuos, junto con los cambios que sufre a lo largo de su vida. Algunos autores, además de incluir en ella a las hierbas, los arbustos y los árboles, mencionan a las enredaderas y a las lianas, o bien únicamente se refieren a plantas erectas, rastreras o trepadoras. De acuerdo con el uso actual del lenguaje, los conceptos de hábito y de forma de crecimiento no pueden separarse en la mayoría de los grupos taxonómicos. Un concepto más amplio es el de 'arquitectura vegetal', el cual considera cuatro aspectos con base en el reconocimiento de las unidades de construcción: el tipo de crecimiento (rítmico o continuo), el patrón de ramificación (monopodial o simpodial), la diferenciación morfológica de los ejes (ortótropa o plagiótropa) y la posición de las estructuras sexuales (terminal o lateral) para asignar un modelo (Barthélémy y Caraglio, 2007; Bell y Bryan, 2008). Estos atributos del estudio arquitectónico pueden ser aplicados a cualquier planta, ya sea que se trate de un árbol, un arbusto, una hierba o una liana (Hallé et al., 1978).

Los miembros de la familia Cactaceae se caracterizan por la morfología peculiar de sus tallos. El hábito en la familia es referido como hábito de crecimiento (Kaplan, 2001) o forma de crecimiento (Buxbaum, 1950; Bravo-Hollis, 1978; Rauh, 1979; Anderson, 2001). En sentido estricto se puede considerar que en la familia Cactaceae hay dos hábitos: arbóreo y arbustivo. Por ejemplo, en la subfamilia Cactoideae se presentan las modificaciones más evidentes de los tallos; cuando el hábito corresponde a un árbol sin ramas es llamado columnar y cuando se ramifica se le conoce como candelabriforme. Cuando la planta se ramifica se distingue en ella un tronco con ramas ortotrópicas (verticales) y paralelas entre sí, como en Pachycereus weberi (J.M.Coult.) Backeb. (Figura 1A) o Stenocereus dumortieri (Scheidw.) Backeb.; la ramificación se simplifica, como en Carnegiea gigantea (Engelm.) Britton \& Rose (Figura 1B), hasta desaparecer, como en Cephalocereus columna-trajani (Karw. ex Pfeiff.) K.Schum. y Stephanocereus leucostele (Gürke) A.Berg. (Figura 1C). En estas dos últimas especies se adquiere la forma de crecimiento columnar (Buxbaum, 1950; Bravo-Hollis, 1978; Anderson, 2001), pero el hábito sigue siendo arbóreo.

El tipo de ramificación en Cactaceae puede ser acrótona cuando las ramas aparecen cerca del ápice del tronco principal, como sucede en Browningia candelaris (Meyen) Britton \& Rose (Figura 1D; Bravo-Hollis, 1978); dicótoma cuando el meristemo apical se divide en dos, como en Hatiora y Rhipsalis (Anderson, 2001); mesótona si de un tronco bien definido salen ramas que se van ramificando sucesivamente hasta formar una copa amplia, como en Myrtillocactus geometrizans (Mart. ex Pfeiff.) Console (Figura 1E), Polaskia chichipe (Gosselin) Backeb. o Pereskia guamacho F.A.C.Weber; o basítona si las ramas surgen desde la base, como en Cephalocereus senilis (Haw.) Pfeiff. (Figura $1 F)$. Las especies con ramificación acrótona o mesótona corresponderían a árboles, sin importar la altura de las plantas, mientras que cuando la ramificación es basítona corresponderían a arbustos.

En plantas de hábito arbustivo todas las ramas surgen cerca del nivel del suelo (ramificación basítona) como en Leptocereus quadricostatus (Bello) Britton \& Rose, Neoraimondia arequipensis (Meyen) Backeb. o Stenocereus stellatus (Pfeiff.) Riccob. (Figura 1G). En las plantas con hábito arbustivo las ramas pueden ser decumbentes, como en $P e$ niocereus greggii (Engelm.) Britton \& Rose o S. gummosus (Engelm. ex Brandegee) A.C.Gibson \& K.E.Horak (Figura 1H); postradas, como en Haageocereus albispinus (Akers) Backeb. y S. eruca (Brandegee) A.C.Gibson \& K.E.Horak (Figura 1I), especie cuyas ramas parecen desplazarse por el suelo (Bravo-Hollis, 1978; Anderson, 2001); o péndulas, como en algunas especies de Disocactus y Selenicereus (Anderson, 2001). Cuando en la familia Cactaceae el hábito es arborescente o arbustivo, como se ejemplificó en los párrafos anteriores, la asignación de estos términos no es difícil; sin embargo, hay tribus como Cacteae donde la asignación del hábito no es fácil.

En la tribu Cacteae, sujeto de este estudio, predominan los géneros con individuos de talla baja, como Aztekium y Mammillaria, pero también hay géneros cuyos integrantes tienen alturas de más de $1 \mathrm{~m}$, como Echinocactus y Ferocactus, o son de tallas intermedias $(20-30 \mathrm{~cm})$, como Escobaria, Neolloydia y Thelocactus. ¿Podrían aplicarse los conceptos de hábito arbóreo (árbol) y herbáceo (hierba) a los miembros de la tribu?, ¿son arbustos algunos miembros de los géneros Acharagma, Mammillaria y Thelocactus? Anderson (2001) señala que en la tribu Cacteae las formas de crecimiento son cespitosas, globosas, globoso-deprimidas o con forma de barril. Algunas de las formas descritas por Anderson (2001) no podrían ser aplicadas en sentido estricto a la forma de crecimiento en una cactácea; por ejemplo, la forma de crecimiento cespitosa. El término 'cespitoso' también se ha utilizado para otras familias como Aizoaceae y Crassulaceae (Eggli y Newton, 2004) con tallos suculentos. Este término entonces se refiere a la ramificación pero no a la forma de crecimiento, como puede notarse en las descripciones de Benson (1982), quien no reconoce la forma de crecimiento cespitosa. El otro término empleado en la literatura de cactáceas se refiere a la forma de crecimiento que en términos coloquiales se define como barril (Bravo-Hollis, 1978; Gibson y Nobel, 1986; Bravo-Hollis y Sánchez-Mejorada, 1991; Anderson, 2001), el cual alude a una forma cilíndrica con las variantes subcilíndrica o ligeramente cilíndrica. ¿Cuáles serían los términos a utilizar para evitar ambigüedad? Entonces, las preguntas a responder en este estudio fueron: ¿cuántas formas de crecimiento hay en Cacteae?, ¿qué nombre deben recibir estas formas? Por lo tanto, nuestro objetivo fue caracterizar las formas de crecimiento dentro de la tribu Cacteae, estableciendo una distinción entre los conceptos de hábito y de forma de crecimiento. 


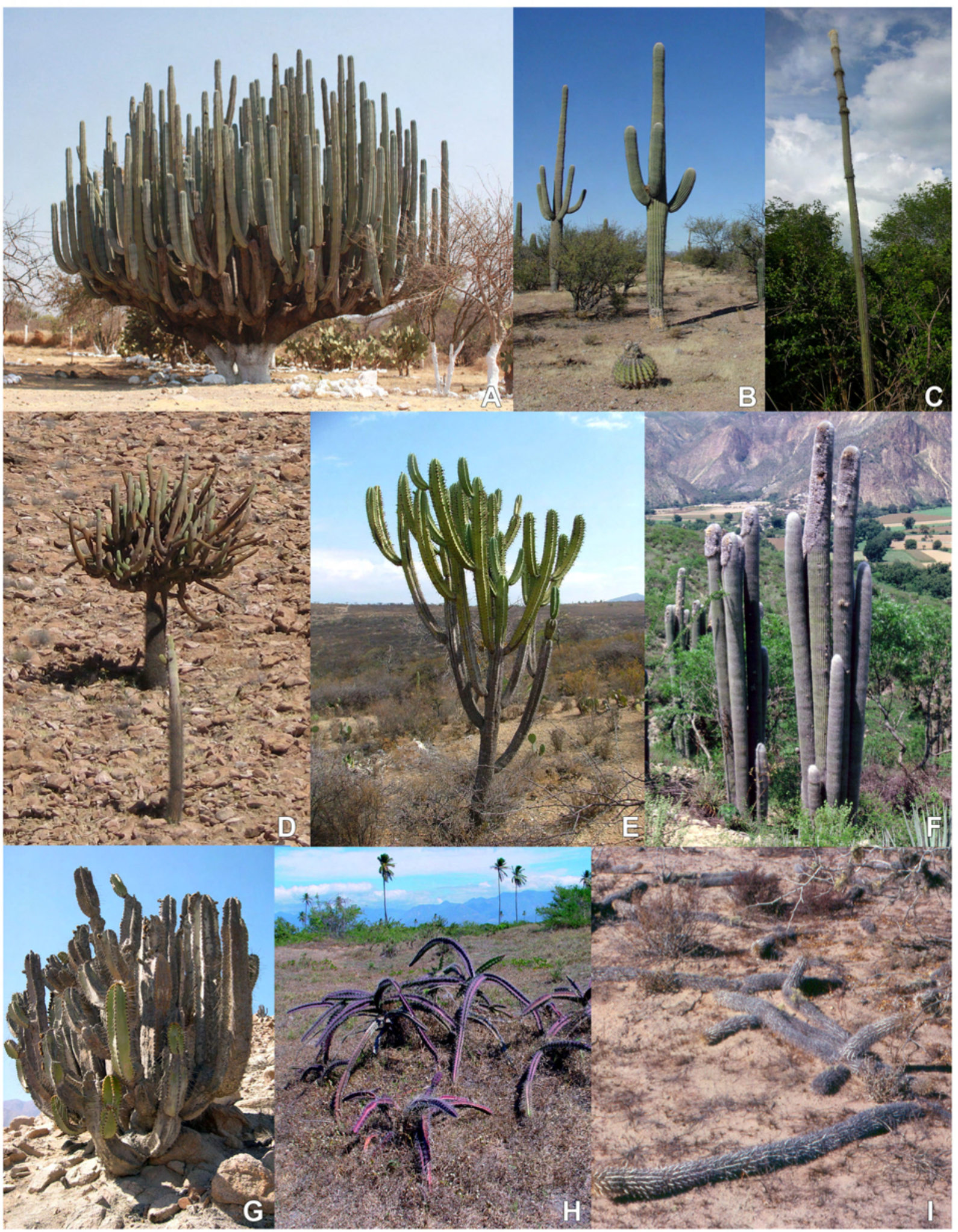

Figura 1. Hábito y tipo de ramificación de algunas especies de Cactoideae pertenecientes a las tribus Browningieae, Cereeae y Pachycereeae. A. Pachycereus weberi (árbol, ramificación mesótona). B. Carnegiea gigantea (árbol, ramificación mesótona). C. Stephanocereus leucostele (árbol, columnar). D. Browningia candelaris (árbol, ramificación acrótoma). E. Myrtillocactus geometrizans (árbol, ramificación mesótona). F. Cephalocereus senilis (arbusto, ramificación basítona). G. Neoraimondia arequipensis (arbusto, ramificación basítona). H. Stenocereus gummosus (arbusto, ramificación basítona, decumbentes). I. Stenocereus eruca (arbusto, basítona, postrado). 


\section{Materiales y métodos}

Se realizó una revisión exhaustiva de la literatura sobre las formas de crecimiento para los miembros de Cacteae tanto en floras como en monografías y descripciones originales.

Para un total de 102 especies se hicieron observaciones y mediciones en campo en las áreas de distribución natural de las especies y éstas se complementaron para algunas especies con observaciones en la colección del Jardín Botánico del Instituto de Biología de la Universidad Nacional Autónoma de México. Para cada especie, por lo menos tres individuos fueron medidos en su altura y diámetro para categorizar las formas de crecimiento. Como parte del estudio para caracterizar su anatomía vascular (Vázquez-Sánchez y Terrazas, 2011; T. Terrazas, datos no publicados) se recolectaron entre una y tres plantas por especie, lo que permitió confirmar si el crecimiento era agregado (varios individuos creciendo juntos) o ramificado. Si la planta era ramificada se asignó el tipo de ramificación (basítona, mesótona, acrótona).

\section{Resultados}

Con base en las observaciones y mediciones efectuadas se establecieron cuatro formas de crecimiento para Cacteae. (1) Globosa, si el tallo tiene aproximadamente la misma altura que diámetro (Figura 2A-C); esta forma de crecimiento se presentó en $24 \%$ de las especies. (2) Globosa-deprimida, si el diámetro del tallo es mayor que su la altura y el eje vertical está comprimido (Figura 2D, E); se presentó en $26 \%$ de las especies. (3) Cilíndrica, crece más en altura que en diámetro, pero la altura no alcanza más del doble que su diámetro; se presentó en $41 \%$ de las especies estudiadas y es común en varios géneros (Figura 2F-H). (4) Columnar, si la altura de la planta es de dos, tres o hasta más de cinco veces el tamaño de su diámetro (Figura 2I); se encontró sólo en 9\% de las especies. Las formas de crecimiento asignadas a las 102 especies de la tribu Cacteae estudiadas se sintetizan en el Cuadro 1. La ramificación se presentó en $12 \%$ de las especies estudiadas; ésta fue basítona en casi todas las especies y mesótona sólo en Mammillaria prolifera.

\section{Discusión}

A pesar de que se ha estudiado la arquitectura de los tallos en Cactaceae y ésta se señala como simple por tener pocas unidades de construcción (Loup, 1983), la delimitación de las formas de crecimiento no ha logrado consenso. Hunt (1989) considera en su concepto de hábito o forma de crecimiento aspectos no sólo de la apariencia general de la planta, sino que incorpora una descripción total de la morfología (p. ej., presencia de hojas, posición de la aréola y número de costillas). Estas características no son comparables entre los diferentes miembros de la familia y podrían considerarse elementos de la arquitectura de cada especie (von Willert et al., 1990; Barthélémy y Caraglio, 2007). Por su parte, Innes y Glass (1991) proponen ocho formas que resumen la propuesta de Hunt, creando subcategorías. Con base en nuestros resultados consideramos que para los miembros de la tribu Cacteae se reconocen cuatro formas de crecimiento.

Los caracteres de la arquitectura de los tallos, del hábito, de las formas de crecimiento y de las formas de vida se han usado indistintamente en los análisis filogéneticos para hacer interpretaciones sobre la evolución de la forma de crecimiento y el hábito. Por ejemplo, Hernández-Hernández et al. (2011) señalan que la forma 'globosa solitaria' se adquirió tempranamente en la diversificación de la tribu y que 'globoso-cespitoso o agrupado' predomina en tres clados, mientras que Nyffeler (2002), con cuatro representantes de Cacteae, señala que la tribu es "cilíndrica corta a globosa o globosa-cespitosa”. Sin embargo, nuestros resultados indican que la forma de crecimiento más común es la cilíndrica y que el uso del hábito en Cacteae es controversial (ver abajo); por ello, las hipótesis de estos autores tienen que confirmarse utilizando los términos adecuados, por ejemplo, las formas de crecimiento aquí propuestas.

El hábito. Los términos 'árbol' y 'arbusto' se han empleado para describir a los miembros de las cuatro subfamilias de Cactaceae: Cactoideae, Maihuenioideae, Opuntioideae y Pereskioideae (Leuenberger, 1986; Anderson, 2001; Taylor y Zappi, 2004; Edwards y Donoghue, 2006). Ambos hábitos tienen en común características como ser leñosos (con crecimiento secundario) y perennes, pero se diferencian en su altura y el tipo de ramificación (Font Quer, 1977; Hickey y King, 2000). En la tribu Cacteae (Cactoideae), a pesar de que todas sus especies tienen crecimiento secundario y son perennes, por su altura, reducción en la ramificación (monopódicos) y suculencia no se les asigna el término 'árbol'. Es importante mencionar que algunos autores asignan el término 'hierba' o 'hierba suculenta' a los miembros de Cactoideae con tallos cortos, globosos y suculentos (Kiesling, 1999; Albesiano y Fernández-Alonso, 2006; Kiesling et al., 2008). Una hierba en sentido estricto es una planta con tallos no lignificados o apenas lignificados (no leñosos) y que decae anualmente (raramente perenne) (Font Quer, 1977; Lincoln et al., 1996). Los miembros de Cacteae no se apegan a esta definición porque son plantas de lento crecimiento y de ciclo de vida relativamente largo (Godínez-Álvarez et al., 2003), tienen abundante tejido secundario (> 60\% de su volumen) en las especies de baja altura (Vázquez-Sánchez y Terrazas, 2011) y son perennes (longevas); por ello no es apropiado considerarlos hierbas.

Es común en la literatura de cactáceas encontrar el término 'cespitoso' cuando surgen tallos (ramas) pequeños en la base de la planta, como por ejemplo en Coryphantha macromeris, o en la parte media del tallo principal o de otra rama, como en Mammillaria prolifera (Craig, 1989; Dicht 


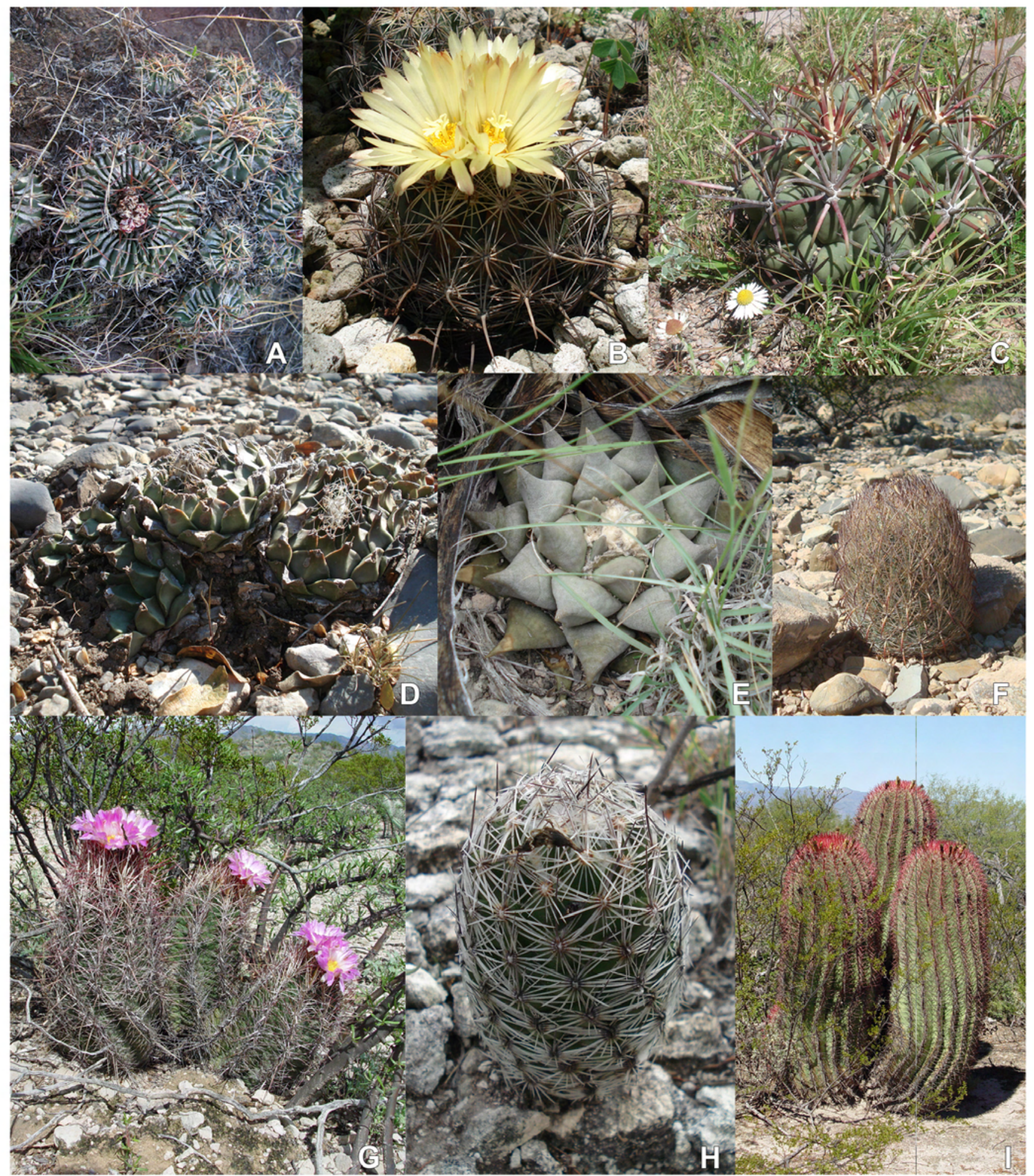

Figura 2. Formas de crecimiento y ramificación de algunas especies de Cacteae. A-C. Globosa. A. Stenocactus crispatus. B. Coryphantha cornifera. C. Glandulicactus crassihamathus. D, E. Globosa-deprimida. D. Obregonia denegrii. E. Ariocarpus retusus. F-H. Cilíndrica. F. Echinomastus unguispinus. G. Thelocactus bicolor, ramificación basítona. H. Neolloydia conoidea. I. Ferocactus pilosus, columnar, ramificación basítona. 


\section{MonserRat VÁZQuez-SÁnCHEZ ET AL.}

Cuadro 1. Caracterización de las formas de crecimiento en especies de la tribu Cacteae. Nombres aceptados por Guzmán et al. (2003).

\begin{tabular}{|c|c|c|c|}
\hline Especie & Forma de crecimiento & Especie & Forma de crecimiento \\
\hline Acharagma aguirreana & globosa & Ferocactus recurvus & cilíndrica \\
\hline Acharagma roseana & cilíndrica & Ferocactus reppenhagenii & cilíndrica \\
\hline Ariocarpus agavoides & globosa deprimida & Ferocactus robustus & cilíndrica \\
\hline Ariocarpus fissutarus & globosa deprimida & Ferocactus townsendianus & cilíndrica \\
\hline Ariocarpus kotschoubeyanus & globosa deprimida & Ferocactus viridescens & globosa \\
\hline Ariocarpus retusus & globosa deprimida & Ferocactus wislizenii & columnar \\
\hline Ariocarpus retusus subsp. trigonus & globosa deprimida & Ferocactus wislizenii subsp. herrerae & columnar \\
\hline Ariocarpus scaphirostris & globosa deprimida & Geohintonia mexicana & globosa \\
\hline Astrophytum asterias & globosa deprimida & Glandulicactus crassihamathus & globosa \\
\hline Astrophytum capricorne & cilíndrica & Glandulicactus uncinatus & cilíndrica \\
\hline Astrophytum myriostigma & cilíndrica & Leuchtenbergia principis & cilíndrica \\
\hline Astrophytum ornatum & cilíndrica & Lophophora diffusa & globosa deprimida \\
\hline Aztekium ritteri & globosa deprimida & Lophophora williamsii & globosa deprimida \\
\hline Cochemiea halei & cilíndrica & Mammillaria albilanata subsp. tegelbergiana & cilíndrica \\
\hline Cochemiea poselgeri & cilíndrica & Mammillaria compresa & globosa \\
\hline Coryphantha cornifera & globosa & Mammillaria elongata & cilíndrica \\
\hline Coryphantha erecta & cilíndrica & Mammillaria heyderi & globosa deprimida \\
\hline Coryphantha georgii & cilíndrica & Mammillaria lenta & cilíndrica \\
\hline Coryphantha macromeris & cilíndrica & Mammillaria prolifera & cilíndrica \\
\hline Coryphantha potosiana & globosa & Mammillaria scrippsiana & globosa \\
\hline Digitostigma caput-medusae & cilíndrica & Mammillaria senilis & cilíndrica \\
\hline Echinocactus grusonii & globosa & Mammillaria uncinata & globosa deprimida \\
\hline Echinocactus horizonthalonius & globosa deprimida & Mammillaria winterae & globosa deprimida \\
\hline Echinocactus parryi & cilíndrica & Mammillaria zephyranthoides & globosa deprimida \\
\hline Echinocactus platyacanthus & columnar & Mammilloydia candida & cilíndrica \\
\hline Echinocactus texensis & globosa deprimida & Neolloydia conoidea & cilíndrica \\
\hline Echinomastus intertextus & cilíndrica & Obregonia denegrii & globosa deprimida \\
\hline Echinomastus mariposensis & cilíndrica & Ortegocactus macdougallii & globosa \\
\hline Echinomastus unguispinus & cilíndrica & Pelecyphora aselliformis & globosa deprimida \\
\hline Epithelantha micromeris & globosa & Pelecyphora strobiliformis & globosa deprimida \\
\hline Escobaria dasycantha & cilíndrica & Sclerocactus scheeri & globosa \\
\hline Escobaria laredoi & globosa & Stenocactus coptonogonus & globosa deprimida \\
\hline Escobaria missouriensis & globosa deprimida & Stenocactus dichroacanthus & globosa \\
\hline Ferocactus alamosanus & columnar & Stenocactus heteracanthus & globosa \\
\hline Ferocactus chrysacanthus subsp.grandiflorus & columnar & Stenocactus multicostatus & globosa deprimida \\
\hline Ferocactus cylindraceus & columnar & Stenocactus pentacanthus & globosa \\
\hline Ferocactus echidne & globosa & Stenocactus phyllacanthus & globosa \\
\hline Ferocactus Emoryi & cilíndrica & Strombocactus disciformis & globosa \\
\hline Ferocactus flavovirens & cilíndrica & Thelocactus bicolor & cilíndrica \\
\hline Ferocactus fordii & cilíndrica & Thelocactus conothelos & globosa \\
\hline Ferocactus glaucescens & globosa & Thelocactus hastifer & cilíndrica \\
\hline Ferocactus gracilis & columnar & Thelocactus heterocromus & globosa deprimida \\
\hline Ferocactus haematacanthus & cilíndrica & Thelocactus hexaedrophorus & globosa deprimida \\
\hline Ferocactus hamatacanthus & cilíndrica & Thelocactus leucacanthus & cilíndrica \\
\hline Ferocactus histrix & cilíndrica & Thelocactus rinconensis subsp. hintonii & cilíndrica \\
\hline Ferocactus latispinus & globosa deprimida & Thelocactus tulensis & cilíndrica \\
\hline Ferocactus macrodiscus & globosa deprimida & Turbinicarpus beguinii & cilíndrica \\
\hline Ferocactus peninsulae & columnar & Turbinicarpus horripilus & cilíndrica \\
\hline Ferocactus pilosus & columnar & Turbinicarpus nieblae & globosa \\
\hline \multirow[t]{2}{*}{ Ferocactus rectispinus } & cilíndrica & Turbinicarpus saueri subsp. knuthianus & globosa \\
\hline & & Turbinicarpus schmiedickeanus subsp. schwarzii & globosa deprimida \\
\hline
\end{tabular}


y Lüthy, 2005). Bravo-Hollis y Sánchez-Mejorada (1991) usan este término para referirse a las cactáceas que son capaces de formar "nuevos brotes en la base o a los lados del tallo". De acuerdo con Font Quer (1977), son plantas cespitosas aquellas que amacollan mucho, crecen muy próximas y llegan a cubrir extensiones más o menos grandes. En un pasto el tallo puede ser erecto o decumbente, y lo más común es que los nuevos brotes de las ramas laterales emerjan del ápice de la hoja envainada. Gibson (2009) señala que el desarrollo de la arquitectura estolonífera y rizomatosa sucede cuando se rompe la punta del brote a través de la vaina, produciéndose así el crecimiento cespitoso. Nuestras observaciones de campo para los miembros de Cacteae mostraron que las ramas nuevas comúnmente surgen de la base del tallo principal o de nuevas ramas, lo cual constituye un caso de ramificación basítona, pero no de un rizoma, como sucede en un pasto, ya que en Cacteae no se ha registrado este tipo de tallo. Consideramos que el término adecuado para referirnos a plantas que tienen esta ramificación es hábito arbustivo. Nuestros resultados indican que $12 \%$ de las especies estudiadas pueden denominarse 'arbustos' porque se ramifican desde la base, como Acharagma roseana, Coryphantha erecta, Ferocactus pilosus, Ortegocactus macdougalli y varias especies de Mammillaria y Thelocactus que tienen diversas formas de crecimiento. Sin embargo, en Airampoa (Opuntioideae), Corryocactus aureus (Meyen) Hutchinson ex Buxb. (Pachycereeae-Cactoideae) y Parodia ottonis (Lehm.) N.P.Taylor (Notocactaceae-Cactoideae) hay ramificaciones laterales, que forman estolones y luego se enraizan (Haustein, 1988) y por ello el uso de término 'cespitoso' sí sería correcto para estas especies con estolones.

La ramificación mesótona de algunas especies de Mammillaria (como M. prolifera), en sentido estricto correspondería a un árbol de talla corta; sin embargo, por su tamaño y cercanía de las nuevas ramas al suelo se le asigna el término arbusto. Un caso excepcional corresponde a la formación de ramas por división del meristemo apical, generando una dicotomía como en Mammillaria morganiana Tiegel, M. muehlenpfordtii Foerster, M. parkinsonii Ehrenb. y M. perbella Hildm. ex K.Schum. (Boke, 1976; Craig, 1989). Este tipo de división del meristemo apical también se presenta en varias especies de Hatiora y Rhipsalis y difiere de la ramificación acrótona, en la que las ramas surgen muy cerca del ápice pero de meristemos laterales.

Con base en los argumentos señalados consideramos que el uso del 'hábito' no es apropiado para las especies de la tribu Cacteae, porque estaríamos forzando su empleo debido a su peculiar morfología (tallas bajas, longevos, suculentos y acumulación de leño escasa). Sin embargo, el hábito si se puede asignar a los miembros de otras tribus como Cereeae y Pachycereeae (Figura 1).

Forma de crecimiento. Con base en nuestros resultados, las formas de crecimiento en Cacteae se reducen a las cuatro figuras geométricas básicas de sus tallos. En general, sus tallos son simples y están limitados a una sola unidad de construcción. Ésta se caracteriza por la abundancia de tejido primario -médula y córtex- (Kaplan y Groff, 1995) y la escasa acumulación de tejido vascular secundario (Altesor et al., 1994; Vázquez-Sánchez y Terrazas, 2011). Además, tienen crecimiento longitudinal indeterminado, el cual permanece activo hasta la muerte de la planta como lo señala Buxbaum (1950) para otras cactáceas, por lo que la forma final que adoptan está definida por su ritmo de crecimiento.

La forma de crecimiento globosa, denominada por Buxbaum (1950) como hábito globoso (forma de esfera, como en Echinocactus grusonii), ya se había reconocido como la forma predominante en la tribu Cacteae (Anderson, 2001). La forma de crecimiento globosa tiene un crecimiento terminal lento y el crecimiento lateral es rápido; una vez que se ha alcanzado el diámetro máximo el crecimiento en altura es muy lento (Buxbaum, 1950). Los individuos con forma de crecimiento globosa pueden encontrarse solitarios o agrupados, dando la apariencia de colonias, como en Stenocactus crispatus (Figura 2A). Otras especies como Acharagma roseana y Ortegocactus macdougallii mantienen la forma de crecimiento globosa y son arbustos por sus ramas basítonas.

La forma de crecimiento denominada globoso-deprimida, es decir, comprimida o con el eje vertical menor que el diámetro del tallo, se caracteriza porque el incremento en diámetro sobrepasa al incremento en altura; se presenta en pocas especies y es la forma de crecimiento característica de las especies de Ariocarpus. Esta forma de crecimiento también ha sido referida por Anderson (2001) como geófita; sin embargo, este término corresponde a una de las formas de vida propuestas por Raunkiaer (1934). Geófita se refiere a plantas cuyos bulbos u órganos de renuevo destinados a sobrevivir en la época desfavorable se sitúan bajo la superficie del suelo o del agua; la profundidad que tienen bajo la superficie varía entre especies (Raunkiaer, 1934). Los tallos y las raíces de muchas especies de Cacteae tienen la capacidad de contraerse durante la época desfavorable, cuando la disponibilidad de agua es baja, como recientemente fue demostrado por Garrett et al. (2010). Esta habilidad de contracción permite a los individuos que crecen al nivel del suelo, como los de las especies de Ariocarpus o de Astrophytum asterias, resistir las sequías extremas a pesar de estar rodeados de suelo que se mantiene mucho más caliente que la temperatura del aire (Geiger et al., 2003). Durante el periodo más desfavorable, ninguna de las especies de la tribu Cacteae aquí estudiadas y que crecen enterradas forman nuevos brotes a partir de su tallo subterráneo. De acuerdo con nuestras observaciones, el uso del término 'geófito' para referirse a la forma de crecimiento en una cactácea que crece a nivel del suelo es inadecuado. La condición geófita es en realidad una estrategia de supervivencia en muchas especies. Las plantas geófitas suculentas (en el sentido de 
Raunkiaer) persisten de un año al siguiente sólo con una parte de sus órganos y lo hacen bajo el suelo, de modo que sobre la superficie se parecen a cualquier planta anual. La mayoría de las plantas suculentas en los desiertos tienen en la superficie su porción vegetativa, que puede no tener hojas (algunas Asclepiadaceae, Cactaceae y algunas Euphorbiaceae) o tener hojas perennes o caducas (von Willert et al., 1990). Sin embargo, esta estrategia de supervivencia no refleja la forma de crecimiento de un tallo.

Cuando los tallos crecen más en altura que en diámetro se origina la forma cilíndrica y es ésta la forma de crecimiento predominante en la tribu Cacteae. En la literatura, a la forma cilíndrica aquí propuesta se le puede encontrar también bajo los términos columnar corta o forma de barril (Gibson y Nobel, 1986; Barthlott y Hunt, 1993; Anderson, 2001). Sin embargo, con fines comparativos recomendamos usar la forma de crecimiento cilíndrica. La característica de esta forma de crecimiento es el incremento rápido en longitud durante los primeros años; una vez que alcanza su diámetro máximo la planta puede seguir creciendo lenta e indeterminadamente, manteniendo la misma proporción en su forma.

La forma de crecimiento de las plantas cuyos tallos alcanzan una altura de más de dos veces su diámetro se definió como columnar. Esta forma de crecimiento se presentó en sólo nueve de las 102 especies, entre ellas Echinocactus platyacanthus y varias especies de Ferocactus como $F$. peninsulae y $F$. townsendianus. La mayoría de las especies con esta forma de crecimiento son monopódicas, excepto $F$. pilosus que tiene ramificación basítona. Esta última especie es un arbusto, sin importar su altura, por su ramificación basítona.

En la literatura de Cactaceae se utiliza el término 'barriliforme' tanto para formas de crecimiento cilíndricas como columnares. Eggli (1993) define la forma barril como "solitary short-columnar, rather than thick stem". Si consideramos estrictamente la definición de barril, entonces sería aquella forma donde la parte media del tallo tiene mayor diámetro que el ápice. Por lo tanto, todos serían barriles y no podríamos discriminar entre especies. Por ello, sugerimos no emplear este término tan amplio y si referirnos a cilíndricos y columnares donde la relación altura-diámetro permite el reconocimiento de las formas de crecimiento.

Forma de crecimiento en el ciclo de vida y relación con su entorno. Las diferentes formas de crecimiento que observamos en Cacteae corresponden a figuras geométricas comunes. La evolución de la morfología de una cactácea seguramente es el resultado de un proceso de selección natural que ha operado para minimizar la ramificación (Price y Enquist, 2006). Sin embargo, debido al crecimiento lento que presentan los miembros de Cacteae podemos encontrar una serie de transformaciones que pueden ser explicadas a la luz de la heterocronía.
La heterocronía, definida por Gould (1977) como la evolución a través de cambios en las tasas de crecimiento o desarrollo, puede producirse por aceleración o retraso del crecimiento (neotenia). La neotenia ha sido utilizada para explicar la variación morfología en el género Sclerocactus. Porter et al. (2000) señalan que los tallos juveniles o neoténicos de Sclerocactus son globoso-deprimidos, mientras que la forma de crecimiento típica de algunas especies en el género es la cilíndrica. Además, sugieren que la neotenia en Sclerocactus se ha adquirido por lo menos en tres ocasiones en forma independiente. En muchas de las especies que aquí se estudiaron las plantas alcanzan la forma de crecimiento característica incluso después de varios eventos reproductivos. Por ello, la neotenia podría estar presente en otras especies, como es el caso de Astrophytum myriostig$m a$, que en sus primeros eventos reproductivos guarda la misma proporción en altura y diámetro, pero en la mayoría de los individuos adultos son cilíndricos. Por lo tanto, es necesario hacer estudios de campo en los que se identifique la heterocronía y ésta se interprete a la luz de la filogenia de Cacteae.

La forma de crecimiento se puede modificar como una respuesta al entorno con ciertos límites ya que está fijada genéticamente (von Willert et al., 1990; Barthélémy y Caraglio, 2007). Por ejemplo, el diámetro de los tallos puede verse afectado por la temperatura o bien las ramas pueden alargarse en áreas donde la vegetación circundante es alta (Racine y Downhower, 1974; Nobel, 1988; Cody, 1984, 1986, 1991; Cornejo y Simpson, 1997). En Cacteae, si la vegetación circundante es más alta puede llevar a que las nuevas ramas que originalmente son globosas se incrementen en longitud hasta tener apariencia cilíndrica, como lo ejemplifican algunas especies de Coryphantha y Mammillaria. Para determinar la forma de crecimiento es necesario entonces tomar en cuenta diferentes factores tanto intrínsecos como extrínsecos de las especies para hacer una correcta caracterización de su forma de crecimiento.

Concluimos que 'hábito' y ‘forma de crecimiento' no son sinónimos para los miembros de Cacteae y es necesario usar estos términos de manera diferenciada para evitar confusiones. En este sentido, se puede hacer la siguiente distinción: 'hábito' describe el aspecto exterior de la planta, es decir, si se trata de un árbol, un arbusto o una hierba, de acuerdo con el concepto de Font Quer (1977), mientras que 'forma de crecimiento' describe la figura geométrica de los individuos. En la tribu Cacteae no es recomendable utilizar el hábito para describir a sus especies porque crea confusión al compararlo con otros miembros de Cactaceae u otras eudicotiledóneas. Sugerimos el uso de las cuatro formas de crecimiento (globosa, globosa-deprimida, cilíndrica, columnar) aquí propuestas para referirnos a los tallos de Cacteae. La asignación de las formas de crecimiento en los miembros Cacteae permitirá entender su evolución al incorporarse a los análisis filogenéticos. 


\section{Agradecimientos}

Se agradece al Posgrado en Ciencias Biológicas de la Universidad Nacional Autónoma de México (UNAM) y al Consejo Nacional de Ciencia y Tecnología (CONACyT) por la beca para realizar estudios de doctorado a MVS (41991), al Programa de Apoyo a Proyectos de Investigación e Innovación Tecnológica (DGAPA, UNAM) por el apoyo a TT (proyecto IN224307), a David Aquino García y Bárbara Larraín por algunas fotografías de campo y a Julio César Montero por diseño gráfico.

\section{Literatura citada}

Albesiano S. y Fernández-Alonso J.L. 2006. Catálogo comentado de la flora vascular de la franja tropical (500-1200 m) del cañón del río Chicamocha (Boyacá-Santander, Colombia), Primera Parte. Caldesiana 28:23-44.

Altesor A., Silva C. y Ezcurra E. 1994. Allometric neoteny and the evolution of succulence in cacti. Botanical Journal of the Linnean Society 114:283-292.

Anderson E.F. 2001. The Cactus Family. Timber Press, Portland.

Barthélémy D. y Caraglio Y. 2007. Plant architectural: a dynamic, multilevel and comprehensive approach to plant form, structure and ontogeny. Annals of Botany 99:375-407.

Barthlott W. y Hunt D.R. 1993. Cactaceae. En: Kubitzki K. Ed. The Families and Genera of Vascular Plants Volume II, pp. 161-197, Springer-Verlag, Berlín.

Bell A.D. y Bryan A. 2008. Plant Form: An Illustrated Guide to Flowering Plant Morphology. Timber Press, Portland.

Benson L. 1982. The Cacti of the United States and Canada. Stanford University Press, Stanford.

Bravo-Hollis H. 1978. Las Cactáceas de México. Vol. I. Universidad Nacional Autónoma de México, México, D.F.

Bravo-Hollis H. y Sánchez-Mejorada H. 1991. Las Cactáceas de México. Vol. III. Universidad Nacional Autónoma de México, México, D.F.

Boke N.H. 1976. Dichotomous branching in Mammillaria (Cactaceae). American Journal of Botany 63:1380-1384.

Buxbaum F. 1950. Morphology of Cacti. Section I. Roots and Stems. Abbey Garden Press, Pasadena.

Cody M.L. 1984. Distribution and morphology of columnar cacti in tropical deciduous woodland, Jalisco, Mexico. Vegetatio 66:137-146.

Cody M.L. 1986. Spacing patterns in Mojave Desert plant communities: near-neighbor analyses. Journal of Arid Environments 11:199-217.

Cody M.L. 1991. Niche theory and plant growth form. Vegetatio 97:39-55.

Cornejo D.O. y Simpson B.B. 1997. Analysis of form and function in North American columnar cacti (tribe Pachycereeae). American Journal Botany 84:1482-1501.

Craig R.T. 1989. The Mammillaria Handbook: with Descriptions, Illustrations, and Key to the Species of the Genus Mammillaria of the Cactaceae. Abbey Garden Press, Pasadena.

Díaz S., Gurvich D.E., Pérez-Harguindeguy N. y Cabido M. 2002. ¿Quién necesita tipos funcionales de plantas? Boletín de la Sociedad Argentina de Botánica 37:135-140.
Dicht R.F. y Lüthy A.D. 2005. Coryphantha: Cacti of Mexico and Southern USA. Springer Verlag, Berlín.

Edwards E.J. y Donoghue M.J. 2006. Pereskia and the origin of the cactus life form. The American Naturalist 167:777-793.

Eggli U. 1993. Glossary of Botanical Terms with Special Reference to Succulent Plants. British Cactus and Succulent Society, Richmond.

Eggli U. y Newton L.E. 2004. Etymological Dictionary of Succulent Plant Names. Springer, Berlín.

Font Quer P. 1977. Diccionario de Botánica. Editorial Labor, Barcelona.

Garrett T.Y., Huynh C-V. y North G.B. 2010. Root contraction helps protect the "living rock" cactus Ariocarpus fissuratus from lethal high temperatures when growing in rocky soil. American Journal of Botany 97:1951-1960.

Geiger R., Aron R.H. y Todhunter P. 2003. The Climate Near the Ground. Rowman and Littlefield Publishers, Nueva York.

Gibson A.C. y Nobel P.S. 1986. The Cactus Primer. Harvard University Press, Cambridge.

Gibson J.D. 2009. Grasses and Grassland Ecology. Oxford University Press, Nueva York.

Godínez-Álvarez H., Valverde T. y Ortega-Baes P. 2003. Demographic trends in the Cactaceae. The Botanical Review 69:173203.

Gould S.J. 1977. Ontogeny and Phylogeny. The Bellknap Press of Harvard University Press, Cambridge.

Guzmán U., Arias S. y Dávila P. 2003. Catálogo de Cactáceas Mexicanas. Universidad Nacional Autónoma de México/Comisión Nacional para el Conocimiento y Uso de la Biodiversidad, México, D.F.

Hallé F., Oldeman R.A.A. y Tomlinson P.B. 1978. Tropical Trees and Forest: An Architectural Analysis. Springer-Verlag, Berlín.

Harris J.G. y Harris. M.W. 2001. Plant Identification Terminology. An Illustrated Glossary. Spring Lake Publishing, Payson.

Haustein E. 1988. The Cactus Handbook. Chartwell Books, Inc., Nueva Jersey.

Hernández-Hernández T., Hernández M.H., De-Nova J.A., Puente R., Eguiarte L.E. y Magallón S. 2011. Phylogenetic relationships and evolution of growth form in Cactaceae (Caryophyllales, Eudicotyledonae). American Journal of Botany 98:44-61.

Hickey M. y King C. 2000. The Cambridge Illustrated Glossary of Botanical Terms. Cambridge University Press, Cambridge.

Holmgren P.K., Keuken W. y Schofield E.K. 2001. Index Herbariorum. International Association for Plant Taxonomy. The New York Botanical Garden, Nueva York.

Hunt D.R. 1989. LVII. Cactaceae. En: The European Garden Flora Committee, Eds. The European Garden Flora, III, pp. 202-301, Cambridge University Press, Cambridge.

Innes C. y Glass C. 1991. Cacti. Portland House, Nueva York.

Judd W.S., Campbell C.S., Kellogg E.A., Stevens P.F. y Donoghue M.J. 2002. Plant Systematics: A Phylogenetic Approach. Sinauer, Sunderland.

Kaplan D.R. 2001. The science of plant morphology: definition, history, and role in modern biology. American Journal of Botany 88: 1711-1741.

Kaplan D.R. y Groff P.A. 1995. Developmental themes in vascular plants: functional and evolutionary significance. En: Hoch P.C. y Stephenson A.G. Eds. Experimental and Molecular Approaches to Plant Biosystematics, pp. 111-145, Missouri Botanical Garden, San Luis. 
Kiesling R. 1999. Cactaceae. En: Zuloaga F.O. y Morrone O. Eds. Catálogo de las Plantas Vasculares de la República Argentina II, p. 423-489, Missouri Botanical Garden, San Luis.

Kiesling R., Faundez L., Larocca J. y Albesiano S.A. 2008. Cactaceae. En: Zuloaga F.O., Morrone O. y Belgrano M. Eds. Catálogo de la Plantas Vasculares del Cono Sur (Argentina, Sur de Brasil, Paraguay y Uruguay) Volumen 2, pp. 1715-1830, Missouri Botanical Garden Press, San Luis.

Leuenberger B.E. 1986. Pereskia (Cactaceae). Memoirs of the New York Botanical Garden No. 41, New York Botanical Garden Press, Nueva York.

Lincoln R.J., Boxshall G.A. y Clark P.F. 1996. Diccionario de Ecologia, Evolución y Taxonomía. Fondo de Cultura Económica, México, D.F.

Loup C. 1993. Premières données sur l'architecture des Cactaceae. Diplôme d'études, Université des Sciences et Techniques du Languedoc, Montpellier. 39 pp.

Moreno N.P. 1984. Glosario Botánico Ilustrado. Instituto Nacional de Investigaciones Sobre Recursos Bióticos, México, D.F.

Mueller-Dombois D. y Ellenberg H. 1974. Aims and Methods of Vegetation Ecology. John Wiley and Sons, Nueva York.

Nyffeler R. 2002. Phylogenetic relationship in the cactus family (Cactaceae) based on evidence from trnK/matK and trnL-trnF sequences. American Journal of Botany 89:312-326.

Nobel P.S. 1988. Environmental Biology of Agaves and Cacti.
Cambridge University Press, Nueva York.

Pavón N.P., Hernández-Trejo H. y Rico-Gray V. 2000. Distribution of plant life forms along an altitudinal gradient in the semi-arid valley of Zapotitlán, México. Journal of Vegetation Science 11:39-42.

Porter J.M., Kinney M.S. y Heil K.D. 2000. Relationship between Sclerocactus and Toumeya (Cactaceae) based on chloroplast trnL-trnF sequences. Haseltonia 7:8-23.

Price C.A. y Enquist B.J. 2006. Scaling of mass and morphology in plants with minimal branching: an extension of the WBE model. Functional Ecology 20:11-20.

Racine C.H. y Downhower J.F. 1974. Vegetative and reproductive strategies of Opuntia (Cactaceae) in the Galápagos Island. Biotropica 6:175-186.

Rauh R. 1979. Kakteen an ihren Standorten. Paul Parey, BerlínHamburgo.

Raunkiaer C. 1934. The Life Forms of Plants and Statistical Plant Geography. Clarendon Press, Oxford.

Taylor N. y Zappi D. 2004. Cacti of Eastern Brazil. Kew Publishing, Kew.

Vázquez-Sánchez M. y Terrazas T. 2011. Stem and wood allometric relationships in Cacteae (Cactaceae) Trees 25:755-767.

von Willert D.J., Eller B.M., Werger M.J.A. y Brinckmann E. 1990. Desert succulents and their life strategies. Vegetatio 90:133-143.

Recibido: 11 de julio de 2011

Aceptado: 6 de febrero de 2012

Editor: Dr. Jorge Meave 


\section{HÁbito y FORMA DE CRECIMIENTO EN CACTEAE}

Apéndice 1. Listado de las especies de Cacteae estudiadas. Las especies se presentan en orden alfabético, con nombre y número de colector. Los ejemplares de respaldo están depositados en MEXU cuando no se menciona el herbario en el texto, o en otros herbarios (acrónimos de acuerdo Holmgrean et al., 2001); en el caso de plantas vivas se muestra el número de accesión del Jardín Botánico de la Universidad Nacional Autónoma de México en seguida del recolector.

Acharagma aguirreana (Glass \& R.A.Foster) Glass, S. Arias 1459; Acharagma roseana (Boed.) E.F.Anderson, C. Glass 6443; Ariocarpus agavoides (Castañeda) E.F.Anderson, H. Sánchez-Mejorada 3628; Ariocarpus fissuratus (Engelm.) K.Schum., S. Arias 1727; Ariocarpus kotschoubeyanus (Lem.) K.Schum., S. Arias 1704; Ariocarpus retusus Scheidw., S. Arias 1720; Ariocarpus retusus subsp. trigonus (F.A.C.Weber) E.F.Anderson \& Fitz Maurice, S. Arias 1993; Ariocarpus scaphirostris Boed., H. Sánchez-Mejorada 3721, S. Arias 1867; Astrophytum asterias (Zucc.) Lem., T. Terrazas 852; Astrophytum capricorne (A.Dietr.) Britton \& Rose, T. Terrazas 892; Astrophytum myriostigma Lem., S. Arias 1730; Astrophytum ornatum (DC.) F.A.C.Weber ex Britton \& Rose, S. Arias 1699b; Aztekium ritterii (Boed.) Boed., S. Arias 1868; Cochemiea halei (Brandegee) Walton, S. Arias 1287; Cochemiea poselgeri (Hildm.) Britton \& Rose, S. Arias 1824; Coryphantha georgii (Scheidw.) Backeb., T. Terrazas 886; Coryphantha cornifera (DC.) Lem., S. Arias 1700; Coryphantha erecta (Lem.) Lem., S. Arias 1684; Coryphantha macromeris (Engelm.) Lem., S. Arias 1788; Coryphantha potosiana (Jacobi) Glass \& R.A.Foster, U. Guzmán 2771; Digitostigma caput-medusae Velazco-Macias \& M.Neváez, S. Arias 1862; Echinocactus grusonii Hildm., J.Z. Ortega s.n.; Echinocactus horizonthalonius Lem., S. Arias 1691; Echinocactus parryi Engelm., S. Arias 1791, Echinocactus platyacanthus Link \& Otto, S. Arias 1679; Echinocactus texensis Hopffer, T. Terrazas 851; Echinomastus intertextus (Engelm.) Britton \& Rose, S. Arias 2032; Echinomastus mariposensis Hester, T. Terrazas 905; Echinomastus unguispinus (Engelm.) Britton \& Rose, S. Arias 1902; Epithelantha micromeris (Engelm.) F.A.C.Weber ex Britton \& Rose, S. Arias 1507; Escobaria dasyacantha (Engelm.) Britton \& Rose, S. Arias 1955; Escobaria laredoi (Glass \& R.A.Foster) N.P.Taylor, S. Arias 1951; Escobaria missouriensis (Sweet) D.R.Hunt, S. Arias 1945; Ferocactus alamosanus (Britton \& Rose) Britton \& Rose, S. Arias 1846; Ferocactus chrysacanthus subsp. grandiflorus (G.E.Linds.) N.P.Taylor, S. Arias 1816; Ferocactus cylindraceus (Engelm.) Orcutt, S. Arias 1808; Ferocactus cylindraceus subsp. tortulispinus (H.E.Gates) N.P.Taylor, S. Arias 1812; Ferocactus echidne (DC.) Britton \& Rose, S. Arias 1682; Ferocactus emoryi (Engelm.) Orcutt, S. Arias 2013; Ferocactus flavovirens (Scheidw.) Britton \& Rose, JB C-12-6-21; Ferocactus fordii (Orcutt) Britton \& Rose, S. Arias 1809; Ferocactus glaucescens (DC.) Britton \& Rose, S. Arias 1701; Ferocactus gracilis H.E.Gates, S. Arias 1810; Ferocactus haematacanthus (Salm-Dyck) Bravo, S. Arias 1796; Ferocactus hamatacanthus (Muehlenpf.) Britton \& Rose, T. Terrazas 828; Ferocactus histrix (DC.) G.E.Linds., S. Arias 1675; Ferocactus latispinus (Haw.) Britton \& Rose, S. Arias 1673; Ferocactus macrodiscus (Mart.) Britton \& Rose, S. Arias 1798; Ferocactus peninsulae (Engelm. ex F.A.C.Weber) Britton \& Rose, S. Arias 1821; Ferocactus pilosus (Galeotti ex Salm-Dyck) Werderm., T. Terrazas 890; Ferocactus rectispinus (Engelm. ex J.M.Coult.) Britton \& Rose, S. Arias 1822; Ferocactus recurvus (Mill.) Y.Ito ex G.E.Linds., S. Arias 1794; Ferocactus reppenhagenii G.Unger, H. J. Arreola 1179, IBUG, Ferocactus robustus (Pfeiff.) Britton \& Rose, S. Arias 1795; Ferocactus townsendianus Britton \& Rose, S. Arias 1825; Ferocactus viridescens (Nutt. ex Torr. \& A.Gray) Britton \& Rose, S. Arias 1801; Ferocactus wislizenii (Engelm.) Britton \& Rose, S. Arias 1789; Ferocactus wislizenii subsp. herrerae (J.G.Ortega) N.P.Taylor, S. Arias 1833; Geohintonia mexicana Glass \& Fitz Maurice, J. Reyes 621; Glandulicactus crassihamathus (F.A.C.Weber) Backeb., S. Arias 1688; Glandulicactus uncinatus (Galeotti ex Pfeiff. \& Otto) Backeb., S. Arias 1899; Leuchtenbergia principis Hook., H. Sánchez-Mejorada 3826; Lophophora diffusa (Croizat) Bravo, S. Arias 35, 1698, 1729; Lophophora williamsii (Lem. ex Salm-Dyck) J.M.Coult., S. Arias 1849; Mammillaria albilanata (H.E.Gates ex G.E.Linds.), S. Arias 1641; Mammillaria compressa DC., T. Terrazas 823; Mammillaria elongata DC., S. Arias 1697; Mammillaria heyderi Muehlenpf., T. Terrazas 829; Mammillaria lenta K.Brandegee, T. Terrazas 907; Mammillaria prolifera (Mill.) Haw., T. Terrazas 884; Mammillaria scrippsiana (Britton \& Rose) Orcutt, S. Arias 1886; Mammillaria senilis G.Lodd. ex Salm-Dyck, S. Arias 1890; Mammillaria uncinata Zucc. ex Pfeiff., S. Arias 1687; Mammillaria winterae Boed., S. Arias 1870; Mammillaria zephyranthoides Scheidw., T. Terrazas 887; Mammilloydia candida (Scheidw.) Buxb., T. Terrazas 885; Neolloydia conoidea (DC.) Britton \& Rose, S. Arias 843; Obregonia denegrii Fric, Zivot \& Prírode, H. Sánchez-Mejorada 3670; Ortegocactus macdougallii Alexander, S. Arias 483; Pelecyphora aselliformis C.Ehrenb., H. Sánchez-Mejorada 3610; Pelecyphora strobiliformis (Werderm.) Fric \& Schelle, H. Sánchez-Mejorada 3844; Sclerocactus scheeri (Salm-Dyck) N.P.Taylor, T. Terrazas 903; Stenocactus coptonogonus (Lem.) A.Berger ex A.W.Hill, U. Guzmán 2770; Stenocactus dichroacanthus (Mart. ex Pfeiff.) A.Berger ex Backeb. \& F.M.Knuth, S. Arias 1758; Stenocactus het- 


\section{MonserRat VÁzQueZ-SÁnchez ET AL.}

Apéndice 1. Continuación

eracanthus (Muehlenpf.) A.Berger ex A.W.Hill, S. Arias 1760; Stenocactus multicostatus (Hildm. ex K.Schum.) A.W.Hill, S. Arias 1774; Stenocactus pentacanthus (Lem.) A.Berger ex A.W.Hill, T. Terrazas 817; Stenocactus phyllacanthus (Mart. ex A.Dietr. \& Otto) A.Berger ex A.W.Hill, T. Terrazas 835; Strombocactus disciformis (DC.) Britton \& Rose, S. Arias 1738; Thelocactus bicolor (Galeotti ex Pfeiff.) Britton \& Rose, T. Terrazas 895; Thelocactus conothelos (Regel \& Klein) Backeb. \& F.M.Knuth, T. Terrazas 844; Thelocactus hastifer (Werderm. \& Boed.) F.M.Knuth, S. Arias 81; Thelocactus heterochromus (F.A.C.Weber) van Oosten, S. Arias 1898; Thelocactus hexaedrophorus (Lem.) Britton \& Rose, T. Terrazas 883; Thelocactus leucacanthus (Zucc. ex Pfeiff.) Britton \& Rose, S. Arias 1678; Thelocactus rinconensis subsp. hintonii Lüthy, S. Arias 1948; Thelocactus tulensis (Poselg.) Britton \& Rose, T. Terrazas 842; Turbinicarpus beguinii (N.P.Taylor) Mosco \& Zanovello, S. Arias 1854; Turbinicarpus horripilus (Lem.) Vác. John \& Riha, J. M. Chalet 204; Turbinicarpus nieblae García-Mor., Mart.-Aval. \& Bergm.Beck., L.G. Martínez 557; Turbinicarpus saueri (Boed.) Vác.John \& Ríha subsp. knuthianus (Boed.) Lüthy, T. Terrazas 882; Turbinicarpus schmiedickeanus subsp. schwarzii (Boed.) Buxb. \& Backeb., T. Terrazas 820; Turbinicarpus valdezianus (H.Moeller) Glass \& R.A.Foster, S. Arias 1853. 